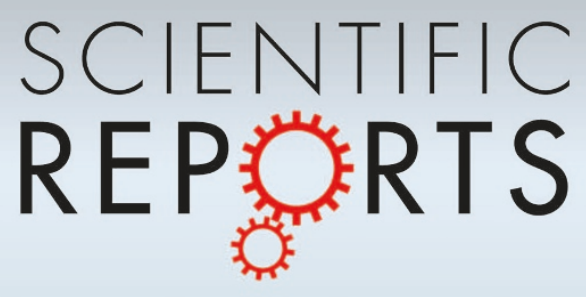

OPEN

SUBJECT AREAS:

TARGETED THERAPIES

APOPTOSIS

OPTICAL MANIPULATION AND

TWEEZERS

NANOTECHNOLOGY IN

CANCER

Received

30 January 2013

Accepted

14 June 2013

Published

5 July 2013

Correspondence and requests for materials should be addressed to

L.M. (minai@bm. technion.ac.il)

\section{High levels of reactive oxygen species in gold nanoparticle-targeted cancer cells following femtosecond pulse irradiation}

\author{
Limor Minai, Daniella Yeheskely-Hayon \& Dvir Yelin
}

Department of Biomedical Engineering, Technion-Israel Institute of Technology, 32000, Haifa, Israel.

Cancer cells could be locally damaged using specifically targeted gold nanoparticles and laser pulse irradiation, while maintaining minimum damage to nearby, particle-free tissue. Here, we show that in addition to the immediate photothermal cell damage, high concentrations of reactive oxygen species (ROS) are formed within the irradiated cells. Burkitt lymphoma B cells and epithelial breast cancer cells were targeted by antibody-coated gold nanospheres and irradiated by a few resonant femtosecond pulses, resulting in significant elevation of intracellular ROS which was characterized and quantified using time-lapse microscopy of different fluorescent markers. The results suggest that techniques that involve targeting of various malignancies using gold nanoparticles and ultrashort pulses may be more effective and versatile than previously anticipated, allowing diverse, highly specific set of tools for local cancer therapy.$$
\text { R }
$$
eactive oxygen species (ROS), a specific type of oxygen-containing reactive molecules, play important roles in various cellular processes, and are known to be essential for cell proliferation at basal levels ${ }^{1}$. At sufficiently high concentrations, however, ROS could become cytotoxic, often entailing cellular necrosis or apoptosis ${ }^{2,3}$, an effect that is frequently utilized for various therapeutic applications. Radiation therapy ${ }^{4}$, i.e. the use of ionizing radiation for treating local malignancies, could damage cells either directly, by ionizing DNA and other cellular molecules, or indirectly, through the production of high levels of ROS that lead to high cytotoxicity. Several chemotherapeutic drugs, for instance Cysplatin ${ }^{5}$ and Actinomycin $D^{6}$, also exploit excess production of ROS for mediating damage to cancer cells ${ }^{5-9}$; while the exact mechanism leading to ROS production using these drugs is not fully understood, it has been shown that the use of ROS scavengers could greatly reduce the drug's effect ${ }^{5,6,8,9}$. ROS also play a key role in photodynamic therapy (PDT), where the activation of a photosensitizer by light initiates a chemical chain reaction which results in local ROS formation ${ }^{10,11}$.

In the last decades, several research groups have proposed the use of specifically targeted noble-metal nanoparticles irradiated by laser light as an effective therapeutic tool for treating various malignancies with minimum collateral damage to healthy tissue ${ }^{12-18}$. Gold nanoparticle-mediated photothermal therapy apply continuouswave $^{13,19-23}$ and pulse laser ${ }^{16-18,24}$ irradiation to induce cellular damage either via extensive temperature rise ${ }^{12,13}$ or through rapid nanoparticle heating and the subsequent release of the energy in the form of small shock waves $^{16-18,24-27}$.

In previous work ${ }^{27}$, our group has demonstrated the induction of selective cell damage with high level of control using gold nanospheres and a few high-intensity femtosecond pulse irradiation. We have shown that resonantly irradiated cells were undergoing either apoptosis, necrosis or were fusing together, depending on cell environment and irradiation parameters (number of pulses, fluence). The exact mechanism that has led to these outcomes, however, is poorly understood, and most likely stemmed from the multiple, nanometric-scale cavitation bubbles which are formed around the irradiated nanoparticles ${ }^{28,29}$. Here, at optical fluence levels below those required for the induction of widespread necrosis, we find that intracellular ROS are formed within Burkitt lymphoma (BJAB) and epithelial breast cancer (MDA-MB-468) cells in concentrations that are proportional to the number of irradiating pulses. The high levels of intracellular ROS could result from the direct interactions between the nanometric shockwaves and the surrounding molecules or indirectly, through the cellular stress caused by these shock waves.

\section{Results}

In order to quantify the formation of ROS in nanoparticle-targeted malignant white blood cells following laser irradiation, Burkitt lymphoma B (BJAB) cells were incubated with 20-nm-diameter gold nanospheres coated by 


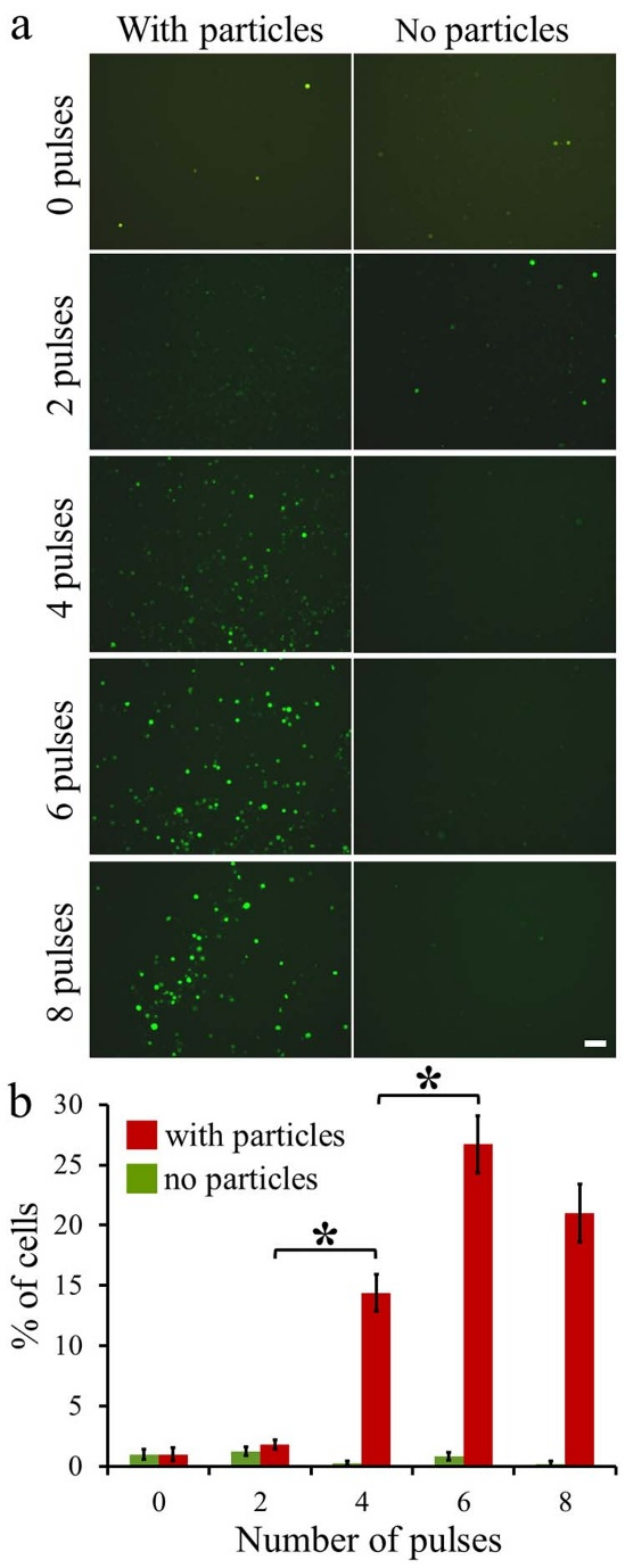

Figure $1 \mid$ ROS in nanoparticle-targeted Burkitt lymphoma cells after irradiation by resonant femtosecond pulses. (a). Fluorescence images of green ROS marker captured 90 minutes after irradiation. Each green spot corresponds to a single cell. Scale bar represents $50 \mu \mathrm{m}$. (b). Percentage of cells in which ROS is higher than the basal level. (*) indicates $\mathrm{P}<0.0001$.

anti-CD20 antibody having high affinity to the membrane-spanning protein CD20 which is localized on the surface of the cell membrane $^{30}$. Following incubation, approximately $10^{4}$ particles were attached to each cell, estimated by measuring the nanoparticle concentration drop in the cell culture medium. Scanning electron microscopy (SEM) has confirmed the presence of gold nanospheres on the cells' plasma membranes (supplementary Fig. 1a). Fluorescence images of cells incubated with $\mathrm{H}_{2} \mathrm{DCFDA}$, a non-fluorescent derivative of fluorescein that is activated upon cleavage by intra-cellular esterases and oxidation, were captured ninety minutes after irradiation by different number of pulses at the resonance wavelength $(550 \mathrm{~nm})$ of the gold nanospheres (Fig. 1a). A bar chart summarizing the percentage of cells expressing ROS levels above the basal level is shown in Fig. 1b. Irradiation by two pulses caused only a minor increase of ROS levels compared to the basal level in cells that were not irradiated and/or not targeted by nanoparticles. Irradiation by four pulses have caused an eight-fold increase in the number of cells containing high cellular ROS, while six pulses have led to high ROS concentrations in nearly $27 \%$ of the cells. The number of cells having high ROS levels after eight pulses was also significantly high, although somewhat lower than after six pulses; we relate this decrease in fluorescence to the expected drop in cell viability due to the strong laser irradiation ${ }^{27}$, which prevented reliable measurement of the intra-cellular ROS levels. The occasional, non-uniform response of the cells across some of the cell cultures is attributed mainly to non-uniformity in the cross-section of the irradiating laser beam, resulting in regions of cells that were irradiated by different pulse intensities. Similar ROS production in irradiated BJAB cells targeted by gold nanoparticles was observed using a different marker of ROS $\left(\right.$ CellROX $\left.{ }^{\mathrm{TM}}\right)$, a cell permeable red dye which fluoresces upon oxidation by ROS (supplementary Fig. 2).

Using time-lapse microscopy of the irradiated BJAB cells, we have found that the majority (approximately 90\%) of cells having ROS concentrations over twice the basal level had eventually died after a few hours. A time sequence of images of a typical region of interest containing three cells with elevated levels of ROS is shown in Fig. 2, depicting the gradual process of cell death. While some of the cells (two cells marked by the two upper arrows) had shown gradual increase in intracellular ROS $\left(\mathrm{H}_{2} \mathrm{DCFDA}\right.$, green) followed by necrosis (red, propidium iodide), others (lower arrow) showed significant swelling ${ }^{31}$, followed by spillage of the cell's content, cell shrinkage and necrosis. By correlating mitochondrial activity (MitoTracker Red CM- $\mathrm{H}_{2}$ XRos) with the distribution of ROS $\left(\right.$ CellROX $\left.{ }^{\mathrm{TM}}\right)$ in irradiated cells, we have found that most of the cells in which elevated levels of ROS were detected were still viable at least two hours post-irradiation (supplementary Fig. 3).

In order to assess the specificity of the process leading to high levels of ROS in irradiated cells, a co-culture (1:1 cell ratio) of CD20-expressing Burkitt lymphoma B cells and K562 erythromyeloblastoid leukemia cells that do not express CD20, was incubated with anti-CD20-coated gold nanoparticles and irradiated by eight pulses. ROS levels in each cell type were measured using the $\mathrm{H}_{2}$ DCFDA marker; the results are shown in supplementary Fig. 4 and summarized in a bar chart in Fig. 3. Approximately $17 \%$ of the Burkitt lymphoma cells in the irradiated co-culture showed elevated levels of ROS, while the ROS levels in the K562 cells remained unaffected. This was consistent with the results of similar experiments using homogeneous K562 cell cultures, in which ROS levels have remained low (Fig. 3) regardless of nanoparticle targeting.

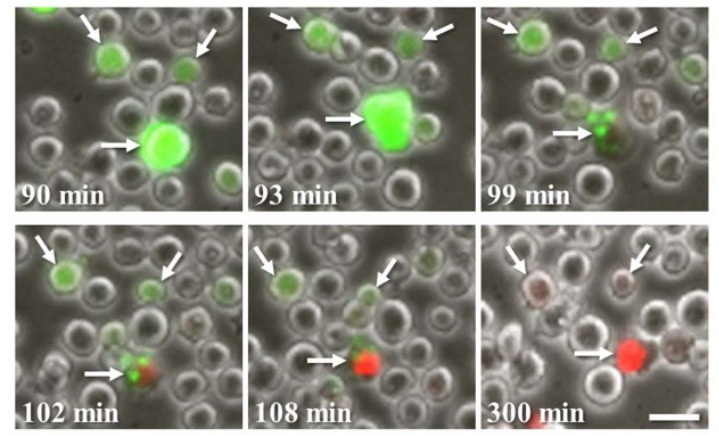

Figure $2 \mid$ Necrosis in Burkitt lymphoma cells following high intracellular levels of ROS, as seen in selected frames from a time-lapse multi-channel fluorescence and phase-contrast microscopy of the nanoparticle-targeted cells irradiated by eight pulses (see lower-left panels in Fig. 1). Numbers at the bottom-left of each frame denote the time elapsed from the moment of irradiation. White arrows point to three representative cells in which excessive ROS (green) is accumulated, followed by necrosis (red). Scale bar represents $20 \mu \mathrm{m}$. 


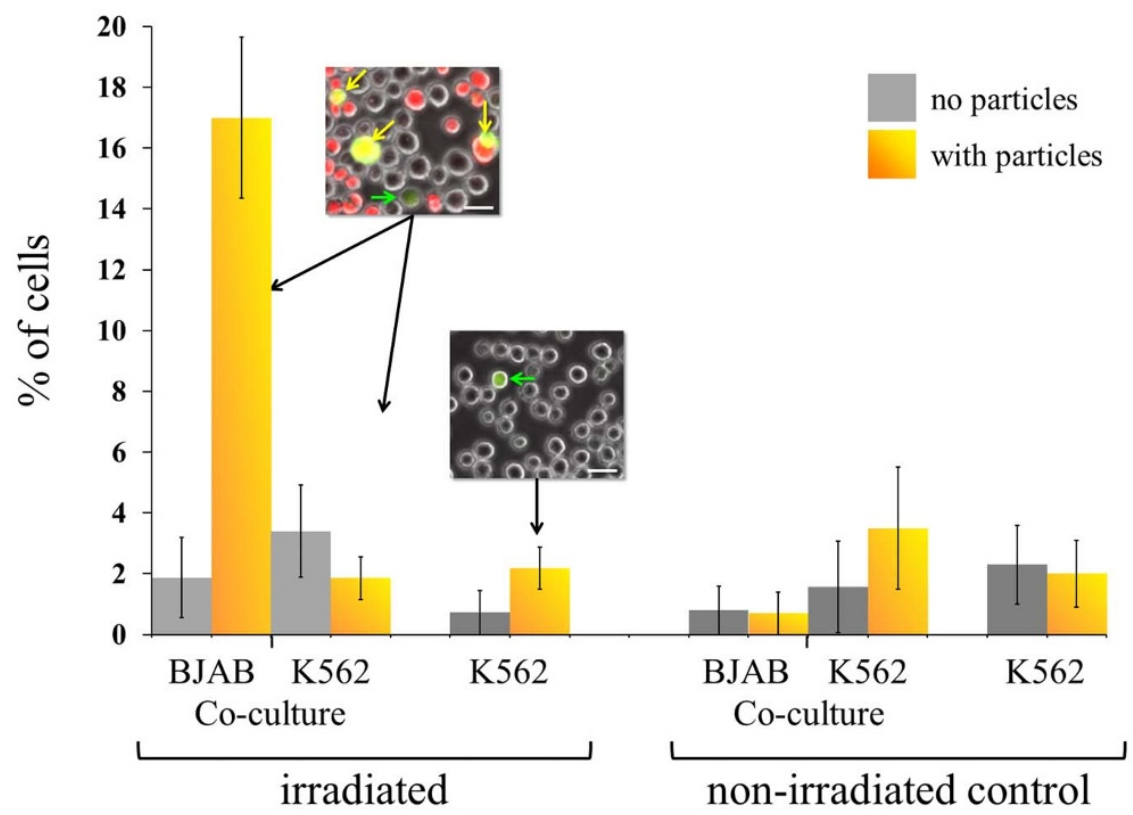

Figure 3 ROS levels in a Burkitt lymphoma (BJAB) and K562 leukemia cell co-culture, and in a homogenous K562 cell culture. The CD20-expressing $\mathrm{BJAB}$ cells respond well to the irradiation, while the K562 cells, that do not express CD20, show no change, either in a co-culture or in a homogeneous culture. Insets show magnified views (original frames are shown in supplementary Fig. 4) from the corresponding fluorescence images of the ROS marker $\mathrm{H}_{2}$ DCFDA (green) and the nucleus marker Hoechst (false color red, BJAB cells only) superimposed on a phase contrast images. Yellow arrows point to BJAB cells with high ROS levels. Green arrows point to K562 cells with high ROS levels. Scale bar represents $40 \mu \mathrm{m}$.

The results presented in Figs. 1-2, showing high concentrations of ROS in irradiated nanoparticle-targeted BJAB cells, were consistent and repeatable throughout numerous experiments, but may be related to the cells' specific chemical or biomechanical properties. Compared to the non-adherent BJAB cells, adherent cells often have different morphology, physiology and mechanical properties, and may respond differently to the combined effect of the laser irradiation and the nanoparticles. In order to study the abundance of ROS in an adherent epithelial MDA-MB-468 cell line, the cells were targeted by anti EGFR-coated gold nanoparticles (approximately $10^{5}$ particles per cell. See also SEM images of a cell membrane in supplementary Fig. 1B) and irradiated by sequences of increasing numbers of femtosecond pulses according to protocols similar to those described above. Fluorescence and phase-contrast images of the epithelial cell cultures (Fig. 4a), and a bar chart summarizing the percentage of cells with high level of cellular ROS (Fig. 4b), reveal significant increase from $5.5 \%$ to $95 \%$ in the number of cells with ROS above the basal level following only two laser pulses. The average increase in fluorescence intensity within the cells was also significant (approximately five-fold), indicating substantial increase of ROS concentrations. After four pulses, a similar percentage of cells with high levels of ROS was observed, however, multinucleated cells have begun to appear as a result of fusion between neighboring cells (cell boundaries marked by dashed yellow curves). After six pulses, the fluorescence level of the ROS marker had dropped significantly, most likely due to decreased cell viability, damage to the plasma membranes, or widespread cell fusion.

When monitored over longer periods of time, the irradiated cells were often losing their viability even when rapid necrosis and/or cell fusion were not immediately observed ${ }^{27}$. Noticeable death of the MDA-MB-468 cells was observed $22 \mathrm{~h}^{32,33}$ post-irradiation even for two irradiation pulses (Fig. 5, top-right panel). While the rate of necrotic cells was similar for all irradiation levels (Fig. 5, red columns), the numbers of apoptotic cells (green columns) were constantly rising in correlation with the number of irradiating pulses. The high levels of intracellular ROS and the absence of immediate visible damage imply that ROS may be the dominant factor ${ }^{2,3}$ in the observed long-term cell damage.

In order to verify that ROS are the main cause of cell damage, cells were incubated with high concentrations of ascorbic acid (vitamin C) $)^{34}$, a well-known antioxidant and an effective scavenger of several reactive oxygen species ${ }^{35}$. At $500 \mu \mathrm{M}$ concentration of ascorbic acid, five-fold higher than typical physiological concentrations, cells have appeared unaffected and ROS levels have remained low even after six irradiation pulses (Fig. 6a, lower-right panel), in contrast to the nearly threefold increase in ROS levels in the absence of ascorbic acid (top-right panel). Approximately fifty hours after irradiation, nearly $24 \%$ of the irradiated culture area was cleared of viable, adherent cells (see Fig. $6 \mathrm{~b}$ for typical regions of interest), while almost full cell coverage was observed in cultures containing ascorbic acid. Approximately 73\% of the irradiated, nanoparticle-targeted cells were counted dead (Fig. 6c, no ascorbic acid), significantly more than the amount (24\%) of dead cells found in irradiated, nanoparticle-targeted cells with ascorbic acid in their growth medium (Fig. 6c).

High levels of intracellular ROS are also known to be the result of Actinomycin D treatment, a common cytotoxic drug whose activity was found to be largely reduced by the presence of ROS scavengers ${ }^{6}$. In order to compare the levels of ROS in nanoparticle-targeted irradiated cells with those induced by Actinomycin D, MDA-MB-468 cells were incubated with Actinomycin D at a concentration known to induce apoptosis in cell cultures ${ }^{36}$. Approximately $92 \%$ of the cells have shown ROS levels significantly higher than the basal level (Fig. 7, see also blue column in Fig. 4b), comparable to the percentage of cells with high levels of ROS following irradiation by two or four pulses. Widespread cell death by apoptosis (Annexin V, green) and necrosis (propidium iodide, red) was evident in these cells five hours after exposure to Actinomycin D (Fig. 7, lower-left panel), comparable to the damage caused to nanoparticle-targeted cells 22 hours after four pulses (see, for example, Fig. 5). A similar trend was observed in BJAB cells following exposure to Actinomycin D (Supplementary Fig. 5). 


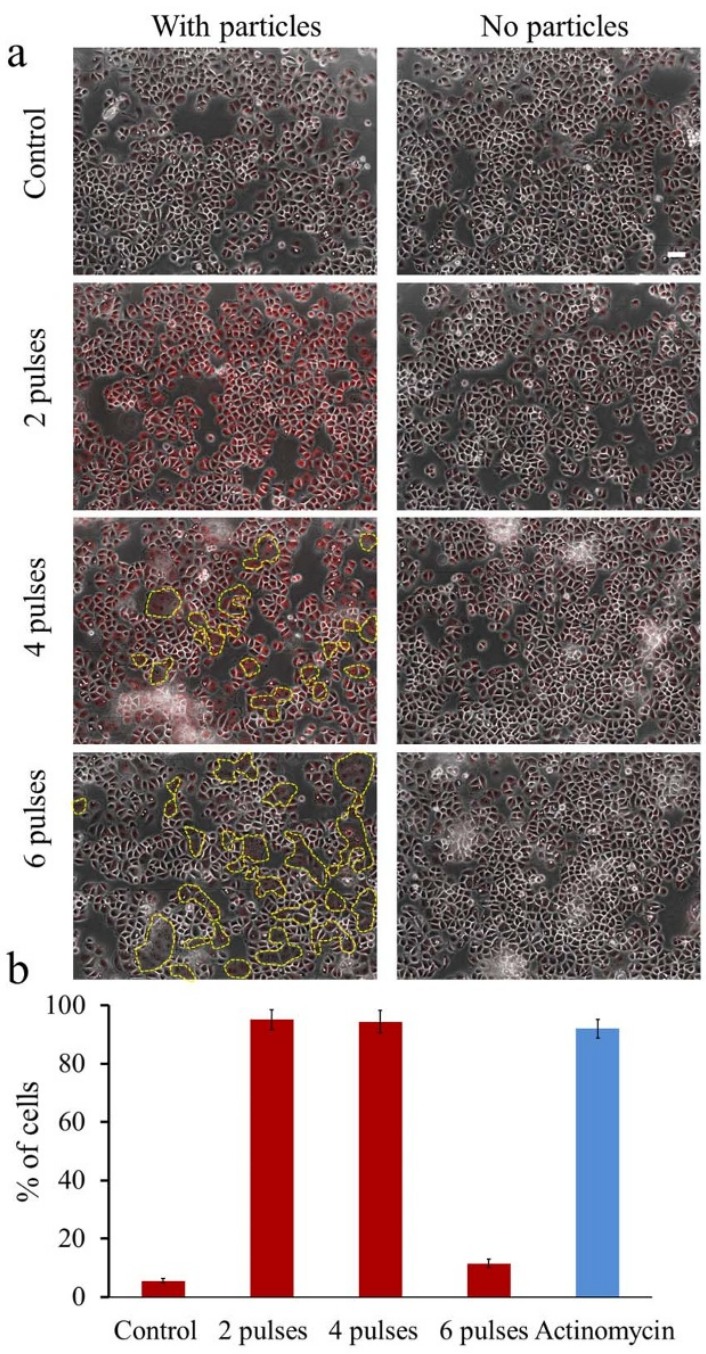

Figure $4 \mid$ ROS in epithelial breast cancer cells following laser pulse irradiation. (a). Fluorescence (red ROS marker) superimposed on phase contrast images of cells irradiated by up to six pulses $\left(25 \mathrm{~mJ} / \mathrm{cm}^{2}\right.$ each). Regions marked by yellow dashed curves represent large multinucleated cells. Scale bar represents $40 \mu \mathrm{m}$. (b). Percentage of cells with ROS concentrations above the basal level. Light blue column: percentage of cells with high levels of ROS following incubation with Actinomycin D.

\section{Discussion}

High power lasers can damage living cells in different ways, most commonly due to elevated tissue temperatures caused by light absorption by the cells. When the laser light is delivered at short pulses of sufficiently high irradiance (near or above $10^{12} \mathrm{~W} / \mathrm{cm}^{2}$ ), multiphoton ionization may lead to (among other processes) excess formation of $\operatorname{ROS}^{37,38}$. Small amounts of ROS could also be produced by light absorption in endogenous porphyrins, mitochondrial cytochromes and flavoproteins ${ }^{39}$. These processes, however, occur whenever laser intensity is sufficiently high, and are often considered nonspecific. By selectively attaching gold nanoparticles to cancer cells and reducing the pulse irradiance below the ionization threshold, specificity is achieved through the local interactions between the pulses and the particles, avoiding damage to nearby, nanoparticlefree cells or tissue.

The current work presents results obtained using two different cell lines: white blood (BJAB) cells with anti CD20-gold nanoparticles targeted to the CD20 proteins on their plasma membranes, and epithelial MDA-MB-468 cells with anti EGFR-gold nanoparticles targeted to the EGFR on their plasma membranes. These specific
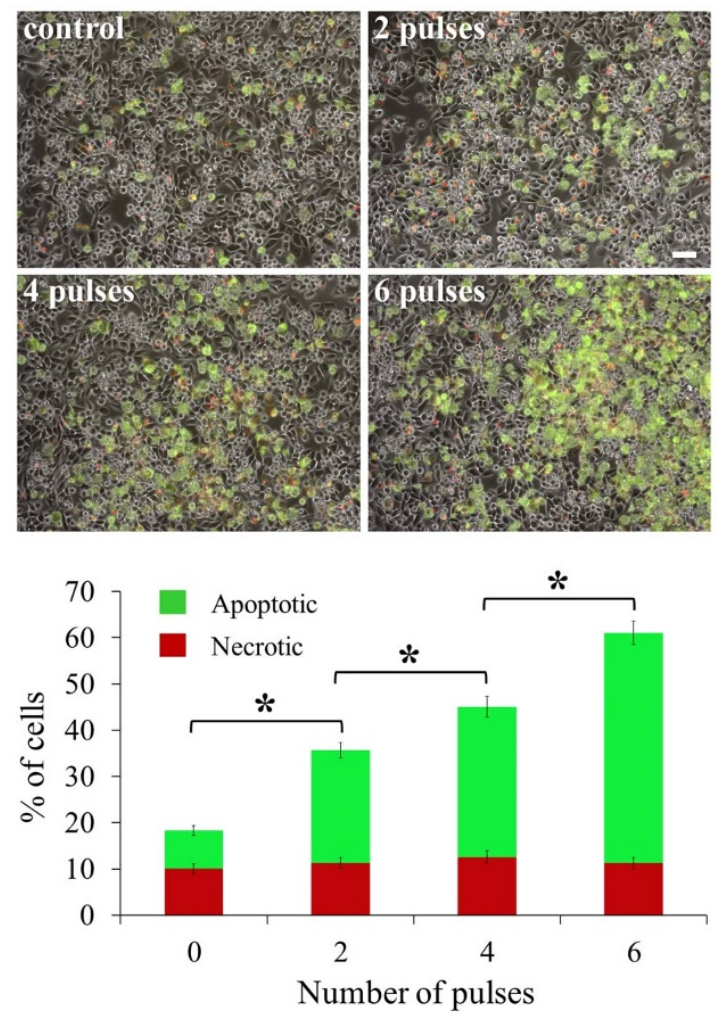

Figure 5 Death of epithelial breast cancer cells as a result of ROS produced by laser pulse irradiation. Red (necrosis) and green (apoptosis) fluorescence distributions superimposed on phase-contrast images, captured $22 \mathrm{~h}$ post irradiation. Irradiation parameters were similar to those described in Fig. 3. Scale bar represents $80 \mu \mathrm{m}$. Bottom bar chart: Percentage of apoptotic (green) and necrotic (red) cells. $\left.{ }^{*}\right)$ indicates $\mathrm{P}<$ 0.0003 .

antibodies were chosen for optimizing targeting efficiency and their contribution to the overall effect of the laser irradiation is expected to be restricted to a minor shift in the plasmonic resonance frequency.

Based on our experimental results, a schematic illustration of the processes leading to high levels of ROS within irradiated nanoparticle-targeted cells is shown in Fig. 8. By irradiating a cell with gold nanoparticles either attached to one (or several) of its plasma membrane receptors, or internalized inside the cell, ROS could be formed as a result of three main processes: 1 . Shock waves generated around the gold nanoparticles due to the high local temperatures and pressure gradients may produce energy densities sufficient for breaking covalent bonds of nearby molecules ${ }^{40-43}$. When generated near nanoparticles that reside outside the cell (e.g. attached to its plasma membrane), ROS molecules may diffuse into the cells through the plasma membrane $e^{44}$ or penetrate through local membrane ruptures caused by the shock wave itself. 2. Multiphoton ionization of molecules within the narrow region around the particles where field enhancement is high ${ }^{45}$ may lead to ROS formation as a result of breakage of covalent bonds. One example would be the breakage of molecular oxygen that generates superoxide and the subsequent production of hydrogen peroxide either outside or inside the cell. Hydrogen peroxide is characterized ${ }^{46,47}$ by long half-life times, sufficient for diffusing through the cells' membranes and residing within the cells over long periods. Experiments with nanoparticle-free cells which were exposed to $50 \mathrm{fs}$ pulses of $1.4 \times 10^{13} \mathrm{~W} / \mathrm{cm}^{2}$, well above the ionization threshold, have shown (see supplementary Fig. 6) high intracellular ROS formation (comparable to those shown in Figs. 1-2), supporting the potential role of multiphoton ionization in the formation of ROS. 3. Cellular stress stemming from the mechanical forces 
no nanoparticles
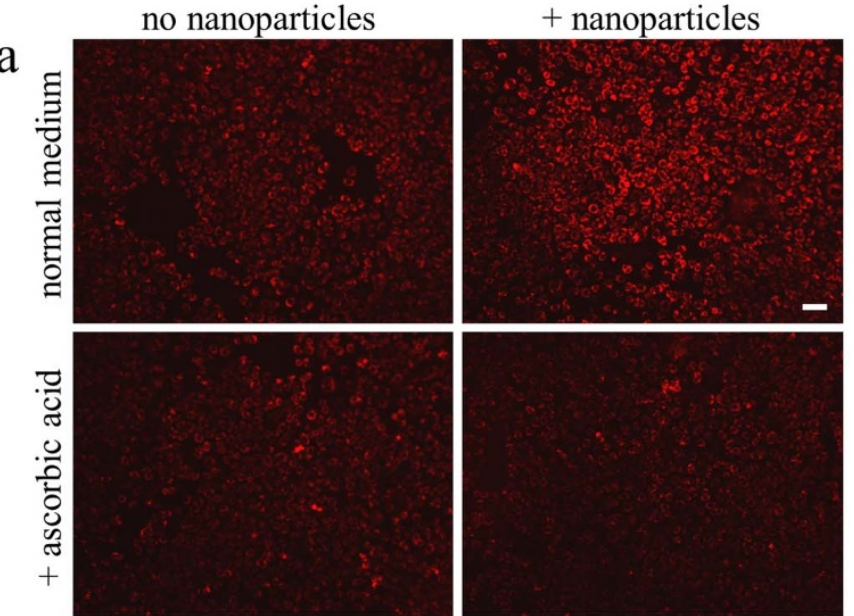

b
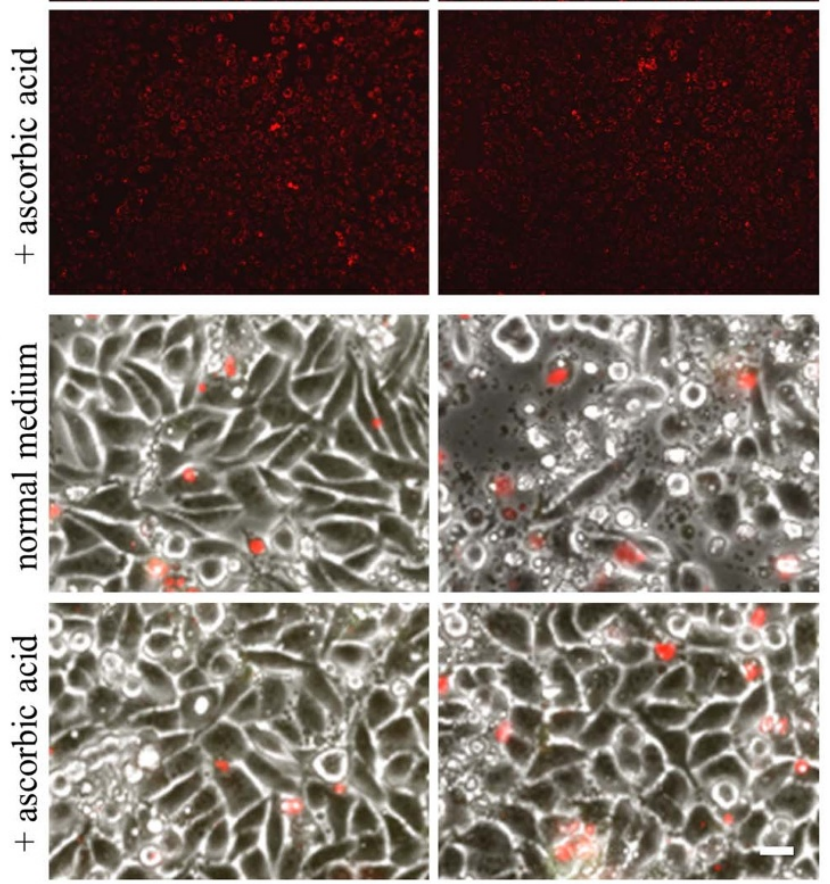

c

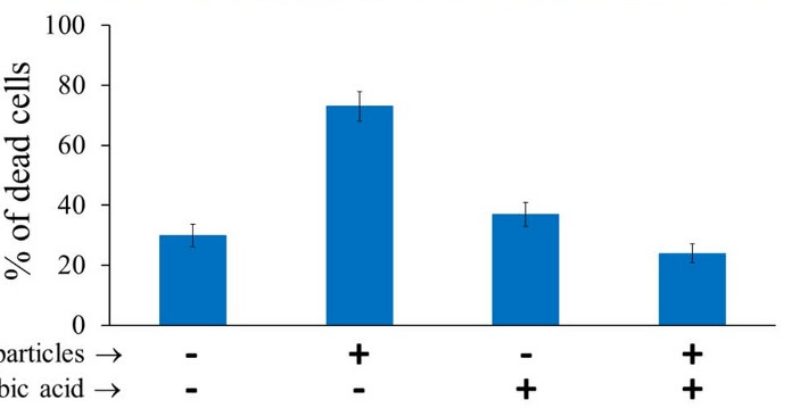

Figure 6 | Ascorbic acid diminishes ROS levels and epithelial breast cancer cells death following irradiation by laser pulses. (a). Fluorescence (Red, CellROX) microscopy of cells $2 \mathrm{~h}$ post irradiation by six pulses (22 $\mathrm{mJ} / \mathrm{cm}^{2}$ each). Scale bar represents $40 \mu \mathrm{m}$. (b). Red (necrosis) fluorescence superimposed on phase-contrast images captured 50 h postirradiation. Scale bar represents $20 \mu \mathrm{m}$. (c). Bar chart representing the percentage of dead cells.

exerted on the cell by the expanding cavitation bubble ${ }^{48,49}$. Being a key player in various cellular stress response processes ${ }^{50}$, ROS may result from the activation of various cellular oxidoreductases which reside in the mitochondria, on the plasma membrane and in the endoplasmic reticulum ${ }^{51}$. Results from experiments designed to study the origin of ROS in irradiated nanoparticle-targeted cells (supplementary Fig. 7) suggest that the mitochondria may not be the source of ROS production following irradiation. Further research would be required for elucidating the relative role of each of these processes in the mechanism that leads to ROS formation under specific experimental conditions.

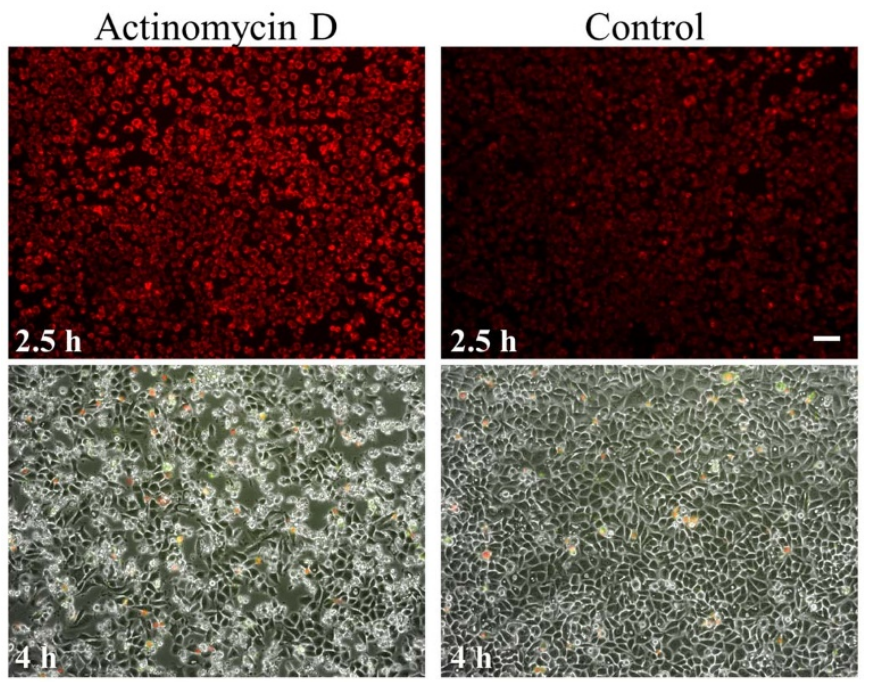

Figure $7 \mid$ ROS and cellular death in epithelial breast cancer cells induced by Actinomycin D. Upper panels: red fluorescence indicates high levels of ROS. Lower panels: red (necrosis) and green (apoptosis) fluorescence distributions superimposed on phase-contrast images. Scale bar represents $80 \mu \mathrm{m}$.

In general, our approach for conveying selective damage to targeted cancer cells, while sparing neighboring untargeted cells, could be exerted through different paths that are briefly summarized in Table 1. After only one or two pulses, high levels of ROS are accumulated within the cells, which could lead to apoptosis within several hours. A higher number of irradiation pulses could lead to additional compromise of the plasma membrane integrity, promoting fusion between neighboring cells ${ }^{27}$. Our results show that these cells also contain high levels of ROS, often leading to their eventual death within several hours. At seven or more laser pulses, ruptures in the plasma membrane often result in rapid cell necrosis, where ROS formation could only accelerate cell death. Finally, the technique presented in this work would be mostly suitable for damaging cells that are accessible to visible light, such as superficial tumors or extracted blood, due to the peak resonance wavelength at $550 \mathrm{~nm}$ of the gold nanospheres that does not penetrate deeply into tissue. The use of antibody-coated gold nanorods with resonance wavelengths at the near infrared would enable deeper penetration into scattering tissues; the efficiency and stability of these particles under intense laser pulse irradiation will be determined in a future study.

In summary, we have shown that cells that were specifically targeted by gold nanoparticles and irradiated by ultrashort laser pulses contain high levels of ROS and consequently dye. This discovery represents an important milestone toward the development of an effective, highly specific cancer treatment, which could be combined with conventional cancer therapies for the treatments of small and/or metastatic malignancies.

\section{Methods}

Cell cultures. BJAB and $\mathrm{K} 562$ cells were grown at $37^{\circ} \mathrm{C}$ and $5 \% \mathrm{CO}_{2}$ in RPMI- 1640 medium (Sigma) and DMEM medium (Invitrogen), respectively, supplemented by $2 \mathrm{mM}$ glutamine, $5 \mathrm{mM}$ sodium pyruvate and $10 \%$ heat-inactivated fetal bovine serum. Cells were maintained at a concentration below $10^{6}$ cells per ml to allow logarithmic growth. MDA-MB- 468 cells were grown at $37^{\circ} \mathrm{C}$ and $5 \% \mathrm{CO}_{2}$ in DMEM medium (Invitrogen) supplemented by $5 \mathrm{mM}$ sodium pyruvate and $10 \%$ heatinactivated fetal bovine serum. For contradicting the effect of ROS, cell cultures were supplemented with fresh medium containing $500 \mu \mathrm{M}$ ascorbic acid two hours prior to irradiation.

Nanoparticle preparation. Gold nanoparticles were prepared using a citrate reduction protocol ${ }^{52}$ resulting in an average particle diameter of $20 \mathrm{~nm}$. Anti-CD20 


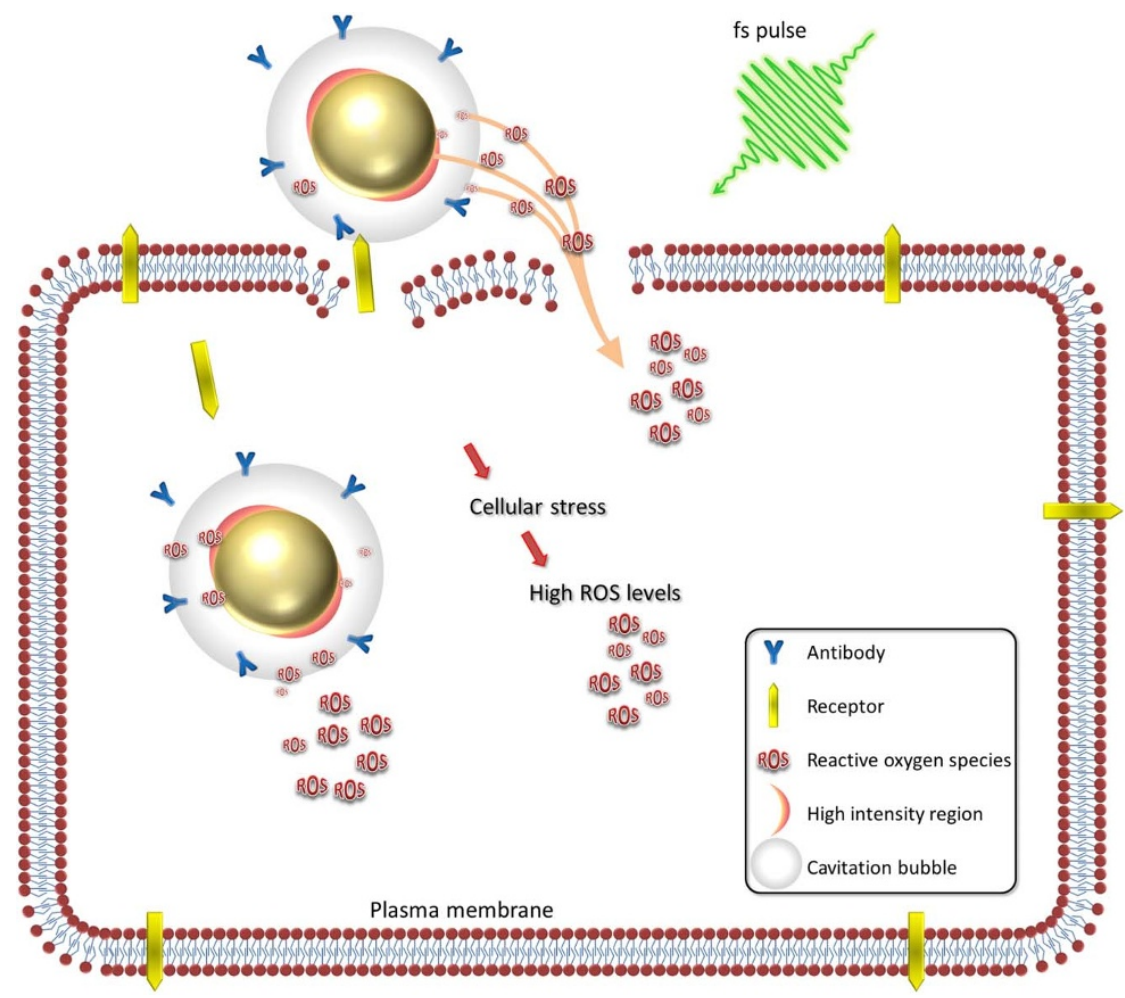

Figure $8 \mid$ Schematic illustration of the proposed model for the processes leading to high levels of ROS in nanoparticle-targeted cells irradiated by femtosecond pulses.

(Rituximab, Roche Israel) coating of the gold nanoparticles was carried out according to Weiss et al..$^{53}$. Anti-EGFR (Lab Vision, clone designation EGFR.1) coating was achieved by incubating anti-EGFR with OPSS-PEG2000-NHS (Jenkem) for $1 \mathrm{~h}$ in a mole ratio of $1: 2500$, followed by additional $1 \mathrm{~h}$ incubation with gold nanospheres in a mole ratio of $1: 10^{6}$ nanospheres: OPSS-PEG2000-NHS

Cell targeting by nanoparticles. BJAB cells $\left(10^{6}\right.$ cells per $\left.\mathrm{ml}\right)$ were incubated for 15 min at $37^{\circ} \mathrm{C}$ with $4 \times 10^{10}$ anti $\mathrm{CD} 20$-coated gold nanoparticles per ml. Cells were washed off unbound gold nanoparticles (three PBS washes) prior to laser irradiation. A co-culture of $1: 1$ ratio BJAB:K562 cells was incubated under the same conditions as described above. MDA-MB-468 cells were incubated for $60 \mathrm{~min}$ at $37^{\circ} \mathrm{C}$ with $2 \times 10^{11}$ anti-EGFR-coated gold nanoparticles per $\mathrm{ml}$. Incubation times for each cell line were set for reaching saturation of the number of nanoparticles attached to each cell.

Fluorescence labeling. In the co-culture experiment, the nuclei of the $\mathrm{BJ} A \mathrm{~B}$ cells were stained blue using $1.3 \mu \mathrm{g} / \mathrm{ml}$ Hoechst (Aldrich) for 4 minutes at $37^{\circ} \mathrm{C}$, followed by 3 PBS washes. Cells were stained for cellular ROS by incubation with $20 \mu \mathrm{M} \mathrm{H}_{2}$ DCFDA (Invitrogen) for $5 \mathrm{~min}$ at $37^{\circ} \mathrm{C}$, or by incubation with $10 \mu \mathrm{M}$ CellROX ${ }^{\mathrm{TM}}$ deep red for $1 \mathrm{~h}$ at $37^{\circ} \mathrm{C}$. Fluorescence microscopy of the ROS levels was conducted approximately $90 \mathrm{~min}$ post-irradiation. The detection of intracellular ROS is highly sensitive to the physiology and metabolism of the cells; hence, different ROS markers that operate through different mechanisms are required for avoiding false positive results. While high levels of ROS in BJAB cells were effectively detected using both $\mathrm{H}_{2}$ DCFDA and CellROX markers, ROS in MDA-MB-468 cells could not be detected by the $\mathrm{H}_{2}$ DCFDA marker, probably due to absence of cellular esterases required for activating fluorescence ${ }^{32,33}$.

Staining for necrosis and apoptosis was carried out using $1 \mu \mathrm{g} / \mathrm{ml}$ Propidium iodide (Sigma) and Annexin V kit (Roche), respectively. Mitochondrial oxidative activity was detected by incubating the cells with $200 \mathrm{nM}$ MitoTracker red (CM$\mathrm{H}_{2} \mathrm{XRos}$; Invitrogen) for $25 \mathrm{~min}$ at $37^{\circ} \mathrm{C}$.
Actinomycin D treatment of MDA-MB-468 cells. Cells were incubated with $10 \mu \mathrm{g} /$ $\mathrm{ml}$ Actinomycin $\mathrm{D}$ for $2 \mathrm{~h}$, followed by three PBS washes.

Laser pulse irradiation. A beam from a Ti:sapphire oscillator (Tsunami, Newport Corp.) was amplified (Spitfire Pro XP) and wavelength-tuned to $550 \mathrm{~nm}$ using an optical parametric amplifier (OPA; Topas-C). Pulse duration was $50 \mathrm{fs}$, at $1 \mathrm{kHz}$ repetition rate. Cells were irradiated within eight-well chamber slides (Lab-Tek II, Thermo-scientific) placed within a microscope stage incubator (Okolab Inc.) at controlled temperature and $\mathrm{CO}_{2}$ concentration. The irradiated area of each cell culture was approximately $100 \mathrm{~mm}^{2}$ and $16 \mathrm{~mm}^{2}$ for the BJAB and MDA-MB-468 cells, respectively, using repeated pulse irradiation at an array of approximately $300 \mu \mathrm{m}$ diameter spots. Single pulse fluence was either $16.5 \mathrm{~mJ} / \mathrm{cm}^{2}\left(3.3 \times 10^{11} \mathrm{~W} /\right.$ $\left.\mathrm{cm}^{2}\right)$ or $24.5 \mathrm{~mJ} / \mathrm{cm}^{2}\left(4.9 \times 10^{11} \mathrm{~W} / \mathrm{cm}^{2}\right)$ for the BJAB and MDA-MB-468 cells, respectively. Multiple pulse irradiations per spot was achieved by tuning the beam scanning rates so that each culture location was irradiated by the desired number of overlapping spots.

Data analysis. Selected images of BJAB and MDA-MB-468 cells, acquired 90 min after irradiation, were used to estimate the percentage of cells with high levels of ROS using NIS-Elements Advanced Research (Nikon) software. The percentage of cells with ROS levels above twice the basal level of the control cells (nanoparticle-free, nonirradiated) was evaluated for different irradiation parameters using manual cell counting. In cultures where cell fusion was abundant, each nucleus within the fused cells was considered as a single ROS-expressing cell, resulting in up to $10 \%$ bias of our estimation toward higher abundance of cells affected by ROS. Selected images of MDA-MB-468 cells, acquired $22 \mathrm{~h}$ and $50 \mathrm{~h}$ post irradiation were used to estimate the percentage of dead (necrotic, apoptotic or cell cadavers) cells for different irradiation parameters using manual counting. The statistical significance of the results was assessed using a two-proportion z-test.

Table 1 | Effect of the number of irradiating pulses on cell fate

\# of pulses

Damage mechanism

$1-2$

$3-6$

7-
ROS

ROS + cell fusion

ROS + cell fusion + membrane rupture
Effect

Apoptosis

Apoptosis, necrosis, multi-nuclei cells

Necrosis 
1. Burdon, R. Superoxide and hydrogen peroxide in relation to mammalian cell proliferation. Free Radical Biology \& Medicine. 18, 775-794 (1995).

2. Fiers, W., Beyaert, R., Declercq, W. \& Vandenabeele, P. More than one way to die: apoptosis, necrosis and reactive oxygen damage. Oncogene. 18, 7719-7730 (1999).

3. Matés, J., Segura, J., Alonso, F. \& Márquez, J. Intracellular redox status and oxidative stress: implications for cell proliferation, apoptosis, and carcinogenesis. Archives of Toxicology. 82, 273-299 (2008).

4. Coia, L. R. \& Moyland, D. J. Introduction to Clinical Radiation Oncology, Medical Physics Publishing, Madison, WI. 1-20 (1998).

5. Bragado, P., Armesilla, A., Silva, A. \& Porras, A. Apoptosis by cisplatin requires p53 mediated p38 $\alpha$ MAPK activation through ROS generation. Apoptosis. 12, 1733-1742 (2007).

6. Ikeda, K. et al. Involvement of hydrogen peroxide and hydroxyl radical in chemically induced apoptosis of HL-60 cells. Biochemical Pharmacology. 57, 1361-1365 (1999).

7. Agostinelli, E. \& Seiler, N. Non-irradiation-derived reactive oxygen species (ROS) and cancer: therapeutic implications. Amino Acids. 31, 341-355 (2006).

8. Block, K. I. et al. Impact of antioxidant supplementation on chemotherapeutic efficacy: A systematic review of the evidence from randomized controlled trials. Cancer Treatment Reviews. 33, 407-418 (2007).

9. Fuchs-Tarlovsky, V. Role of antioxidants in cancer therapy, Nutrition. 29, 15-21 (2013).

10. Lee, S. et al. Pulsed diode laser-based monitor for singlet molecular oxygen. J. Biomed. Opt. 13, 034010-034010 (2008).

11. Celli, J. P. et al. Imaging and Photodynamic Therapy: Mechanisms, Monitoring and Optimization. Chem Rev. 110, 2795-2838 (2010).

12. Hirsch, L. R. et al. Nanoshell-mediated near-infrared thermal therapy of tumors under magnetic resonance guidance. Proc. Nat. Acad. Sci. USA. 100, 13549-13554 (2003).

13. O’Neal, D. P., Hirsch, L. R., Halas, N. J., Payne, J. D. \& West, J. L. Photo-thermal tumor ablation in mice using near infrared-absorbing nanoparticles. Cancer Letts. 209, 171-176 (2004)

14. El-Sayed, I. H., Huang, X. \& El-Sayed, M. A. Selective laser photo-thermal therapy of epithelial carcinoma using anti-EGFR antibody conjugated gold nanoparticles. Cancer Letts. 239, 129-135 (2006).

15. Huang, X. et al. Plasmonic photothermal therapy (PPTT) using gold nanoparticles. Lasers Med Sci 23, 217-228 (2008).

16. Li, J. L., Day, D. \& Gu, M. Ultra-low energy threshold for cancer photothermal therapy using transferrin-conjugated gold nanorods. Adv. Mater. 20, 3866-3871 (2008).

17. Tong, L. et al. Gold nanorods mediate tumor cell death by compromising membrane integrity. Adv Mater. 19, 3136-3141 (2007).

18. Takahashi, H., Niidome, T., Nariai, A., Niidome, Y. \& Yamada, S. Gold nanorodsensitized cell death: microscopic observation of single living cells irradiated by pulsed near-infrared laser light in the presence of gold nanorods. Chem. Letts. 35, 500-501 (2006).

19. Terentyuk, G. S. et al. Laser-induced tissue hyperthermia mediated by gold nanoparticles: toward cancer phototherapy. J. Biomed. Opt. 14, 021016-1-021016-9 (2009).

20. Huang, X. et al. Comparative study of photothermolysis of cancer cells with nuclear-targeted or cytoplasm-targeted gold nanospheres: continuous wave or pulsed lasers. J. Biomed. Opt. 15, 058002-1-058002-7 (2010).

21. Krpeticć, Z et al. Inflicting controlled nonthermal damage to subcellular structures by laser-activated gold nanoparticles. Nano Letts. 10, 4549-4554 (2010).

22. Raji, V. et al. Selective photothermal efficiency of citrate capped gold nanoparticles for destruction of cancer cells. Exp. Cell Res. 317, 2052-2058 (2011).

23. Choi, W. I. et al. Tumor regression in vivo by photothermal therapy based on goldnanorod-loaded, functional nanocarriers. ACS Nano. 5, 1995-2003 (2011).

24. Huang, X., Qian, W., El-Sayed, I. H. \& El-Sayed, M. A. The potential use of the enhanced nonlinear properties of gold nanospheres in photothermal cancer therapy. Lasers in Surgery and Medicine. 39, 747-753 (2007).

25. Pustovalov, V. K., Smetannikov, A. S. \& Zharov, V. P. Photothermal and accompanied phenomena of selective nanophotothermolysis with gold nanoparticles and laser pulses. Laser Phys. Letts. 5, 775-792 (2008).

26. Huff, T. B. et al. Hyperthermic effects of gold nanorods on tumor cells. Nanomedicine. 2, 125-132 (2007)

27. Minai, L. et al. Optical nanomanipulations of malignant cells: controlled cell damage and fusion. Small. 8, 1732-1739 (2012)

28. Volkov, A. N., Sevilla, C. \& Zhigilei, L. V. Numerical modeling of short pulse laser interaction with Au nanoparticle surrounded by water. Applied Surface Sci. 253, 6394-6399 (2007).

29. Nuzzo, V., Maxwell, I., Chung, S., Mazur, E. \& Heisterkamp, A. Subcellular surgery and nanoneurosurgery using femtosecond laser pulses. Biophotonics: Spectroscopy, Imaging, Sensing, and Manipulation. NATO Science for Peace and Security Series B: Physics and Biophysics. Part 1, 203-218 (2011).

30. Valentine, M. A., Cotner, T., Gaur, L., Torres, R. \& Clark, E. A. Expression of the human B-cell surface protein CD20: alteration by phorbol 12-myristate 13 acetate. Proc. Nat. Acad. Sci. USA. 84, 8085-8089 (1987).

31. Jou, M.-J., Jou, S.-B., Chen, H.-M., Lin, C.-H. \& Peng, T.-I. Critical role of mitochondrial reactive oxygen species formation in visible laser irradiation-induced apoptosis in rat brain astrocytes (RBA-1). J. Biomed. Sci. 9, 507-516 (2002).

32. Lizard, G. et al. Kinetics of plasma membrane and mitochondrial alterations in cells undergoing apoptosis. Cytometry. 21, 275-283 (1995).
33. Kanduc, D. et al. Cell death: apoptosis versus necrosis. Int. J. Oncol. 21, 165-170 (2002).

34. Chen, Q. et al. Pharmacologic ascorbic acid concentrations selectively kill cancer cells: Action as a pro-drug to deliver hydrogen peroxide to tissues. Proc. Natl. Acad Sci. USA. 102, 13604-13609 (2005).

35. Fuchs-Tarlovsky, V. Role of antioxidants in cancer therapy. Nutrition. 29, 15-21 (2012).

36. Verhaegen, S., McGowan, A. J., Brophy, A. R., Fernandes, R. S. \& Cotter, T. G. Inhibition of apoptosis by antioxidants in the human HL-60 leukemia cell line. Biochem. Pharmacol. 50, 1021-1029 (1995).

37. Tirlapur, U. K., Konig, K., Peuckert, C., Krieg, R. \& Halbhuber, K.-J. Femtosecond near-infrared laser pulses elicit generation of reactive oxygen species in mammalian cells leading to apoptosis-like death. Exp. Cell Res. 263, 88-97 (2001)

38. King, B. A. \& Oh, D. H. Spatial control of reactive oxygen species formation in fibroblasts using two-photon excitation. Photochem. photobiol. 80, 1-6 (2004).

39. Lubart, R. et al. Low-energy laser irradiation promotes cellular redox activity. Photomedicine and Laser Surgery. 23, 3-9 (2005).

40. Riesz, P., Berdahl, D. \& Christman, C. L. Free radical generation by ultrasound in aqueous and nonaqueous solutions. Environ Health Perspect. 64, 233-252 (1985)

41. Suhr, D., Brummer, F. \& Hulser, D. F. Cavitation-generated free radicals during shock wave exposure: Investigations with cell-free solutions and suspended cells. Ultrasound in Medicine \& Biology. 17, 761-768 (1991).

42. Suslick, K. S. Sonochemistry. Science. 247, 1439-1445 (1990).

43. Huber, P. E. \& Debus, J. Tumor cytotoxicity iIn vivo and radical formation in vitro depend on the shock wave-induced cavitation dose. Radiation. res. 156, 301-309 (2001).

44. Henzler, T. \& Steudle, E. Transport and metabolic degradation of hydrogen peroxide in Chara corallina: model calculations and measurements with the pressure probe suggest transport of $\mathrm{H}_{2} \mathrm{O}_{2}$ across water channels. J. Exp. Botany. 51, 2053-2066 (2000).

45. Bisker, G. \& Yelin, D. Noble-metal nanoparticles and short pulses for nanomanipulations: theoretical analysis. JOSA B. 29, 1383-1393 (2012).

46. Batandier, C., Fontaine, E., Kériel, C. \& Leverve, X. M. Determination of mitochondrial reactive oxygen species: methodological aspects. J. Cell. Mol. Med. 6, 175-187 (2002).

47. Baciocchi, R., Boni, M. R. \& D’Aprile, L. Hydrogen peroxide lifetime as an indicator of the efficiency of 3-chlorophenol Fenton's and Fenton-like oxidation in soils. J. Hazardous Mater. 96, 305-329 (2003).

48. Vogel, A. \& Venugopalan, V. Mechanisms of pulsed laser ablation of biological tissue. Chem. Rev. 103, 577-644 (2003).

49. Rau, K. R., Quinto-Su, P. A., Hellman, A. N. \& Venugopalan, V. Pulsed laser microbeam-induced cell lysis: Time-resolved imaging and analysis of hydrodynamic effects. Biophys. J. 91, 317-329 (2006).

50. Kultz, D. Molecular and evolutionary basis of the cellular stress response. Annu. Rev. Physiol. 67, 225-257 (2005).

51. Jiang, F., Zhang, Y. \& Dusting, G. J. NADPH oxidase-mediated redox signaling: roles in cellular stress response, stress tolerance, and tissue repair. Pharmacol. Rev. 63, 218-242 (2011)

52. Porta, F. et al. Gold nanoparticles capped by peptides. Materials Science and Engineering: B. 140, 187-194 (2007).

53. Weiss, A. et al. Selective recognition of Rituximab-functionalized gold nanoparticles by lymphoma cells studied with 3D imaging. Am. Chem. Soc. 113, 20252-20258 (2009).

\section{Acknowledgments}

The authors wish to thank Lior Golan for his technical assistance, Gili Bisker for helping with the statistical analysis, and Haneen Yameen for her help in preparing the antibody-coated nanoparticles. The research was funded by the European Research Council starting grant (number 239986) and by the Lorry I. Lokey Interdisciplinary Center for Life Sciences and Engineering.

\section{Author contributions}

L.M. has designed and performed the experiments, analysed the data and wrote the manuscript. D.Y.-H. has developed necessary research tools and methods. D.Y. has directed the research.

\section{Additional information}

Supplementary information accompanies this paper at http://www.nature.com/ scientificreports

Competing financial interests: The authors declare no competing financial interests.

How to cite this article: Minai, L., Yeheskely-Hayon, D. \& Yelin, D. High levels of reactive oxygen species in gold nanoparticle-targeted cancer cells following femtosecond pulse irradiation. Sci. Rep. 3, 2146; DOI:10.1038/srep02146 (2013).

This work is licensed under a Creative Commons AttributionNonCommercial-NoDerivs 3.0 Unported license. To view a copy of this license, visit http://creativecommons.org/licenses/by-nc-nd/3.0 